\title{
The role of sublobar resections in the treatment of early stage non-small cell lung cancer-still awaiting evidence
}

\author{
Robert Dziedzic \\ Department of Thoracic Surgery, Medical University of Gdansk, Gdansk, Poland \\ Correspondence to: Robert Dziedzic. Department of Thoracic Surgery, Medical University of Gdansk, 80-210, M. Sklodowskiej-Curie 3a St, Gdansk, \\ Poland. Email: dziedzic@gumed.edu.pl. \\ Provenance: This is an invited Editorial commissioned by Section Editor Dr. Akira Hamada (Department of Cardiovascular, Thoracic and Pediatric \\ surgery, Faculty of Medicine, Yamagata University, Yamagata, Japan). \\ Comment on: Nishio W, Yoshimura M, Maniwa Y, et al. Re-Assessment of Intentional Extended Segmentectomy for Clinical T1aN0 Non-Small Cell \\ Lung Cancer. Ann Thorac Surg 2016;102:1702-10.
}

Submitted Sep 02, 2017. Accepted for publication Sep 16, 2017.

doi: $10.21037 /$ jtd.2017.10.115

View this article at: http://dx.doi.org/10.21037/jtd.2017.10.115

Lobectomy and mediastinal lymphadenectomy remain a standard of care in vast majority of non-small cell lung cancer (NSCLC) patients. According to the European Society for Medical Oncology (ESMO) recommendations, sublobar resections including anatomical segmentectomy and wedge resection may be recommended in highly selected patients, for instance patients with limited respiratory reserves or those with other serious medical conditions, in whom lobectomy would be associated with a significant increase of perioperative risk. Another indication to perform segmentectomy seem to be a peripheral lung tumor with a diameter of less than $2 \mathrm{~cm}$ with a non-solid character or part-solid component $<50 \%$ of the tumor diameter (1). Due to the widespread use of computed tomography in lung cancer screening programs and aging of the population, the number of patients that will be able to meet the above-mentioned criteria probably would increase in the forthcoming years.

But is there sufficient evidence that segmentectomy or wedge resection is equivalent to lobectomy in the treatment of early stage NSCLC in terms of long-term and diseasefree survival?

So far, one randomized study that compared treatment results of lobectomy and sublobar resection has been published. Ginsberg et al. published in 1995 on behalf of Lung Cancer Study Group (LCSG), the long-term results of lobectomy versus sublobar resection, (including segmentectomy and wedge resection) that was performed in the randomized fashion. This study showed that the overall survival of patients after sublobar resection was significantly lower, and the risk of local recurrence was three times higher compared to lobectomy group (2). Despite the limitations of this study, like the enrollment of patients with a tumor diameter of more than $2 \mathrm{~cm}$, nonperipheral pulmonary lesions, wedge resections without lymphadenectomy, lack of appropriate preoperative staging - for many years, lobectomy has become a treatment of choice for the majority of NSCLC patients.

In recent years, the results of numerous observational non-randomized studies comparing lobectomies and sublobar resections have been published. In several observational studies, lobectomy and segmentectomy has shown comparable long-term outcomes in stage I NSCLC less than $2 \mathrm{~cm}$ in diameter $(3,4)$. Most of the studies shows similar outcomes in lobectomy and sublobar resection but there are contradictory results when wedge resection and segmentectomy are analyzed separately. According to Altorki et al. a wedge resection and segmentectomy is associated with comparable outcome for patients with small cT1N0 NSCLC (5). In retrospective observational study of Dziedzic et al., which assessed the results of treatment of stage I NSCLC, wedge resection was associated with significantly worse five-year survival rate compared to lobectomy and segmentectomy (4). What may be the difference between wedge and segmental resection is the resection margin and hilar and mediastinal 
lymphadenectomy. It has been shown that a higher resection margin is associated with a lower percentage of local recurrences. Resection margin greater than $11 \mathrm{~mm} \mathrm{(6)}$ or greater than tumor diameter (7) is a predictor of good survival.

The evidence supporting application of segmentectomy in early stage NSCLC is primarily based on retrospective studies. Currently, there are at least two ongoing prospective, multicenter, randomized trials comparing lobectomies with sublobar resections. The JCOG 0802/ WJOG 4607L study was launched in Japan in 2009 to evaluate the overall survival of patients after segmentectomy and lobectomy for NSCLC (8). There are 71 centers where 1,100 individuals will be recruited. A similar study pending in USA-CALGB 140503-where 692 people are planned to be recruited. Research results are unlikely to be published until 2020 (9).

Theoretically, the extended segmentectomy might be a solution that can help to increase the resection margin of NSCLC during sublobar resection. The Study Group of Extended Segmentectomy for Small Lung Tumor have published a prospective study that evaluated NSCLC treatment results with a diameter of up to $2 \mathrm{~cm}$ using the so called extended segmentectomy (10). The extended segmentectomy technique is based on the inflation of the lung segment before the bronchus is resected, which allows visualization and resection at the border of adjacent lung segments. Moreover, the procedure includes lymphadenectomy of the hilar and mediastinal lymph nodes, similar to that during lobectomy. The reported 5 -year disease-free survival was $91.8 \%$, and the postoperative respiratory volume loss was $11.3 \%$ in the forced vital capacity (FVC) and $13.4 \%$ in the forced expiratory volume in first second (FEV 1). The authors concluded that extended segmentectomy is a suitable type of surgery for patients with peripheral lung tumors with diameter less than $2 \mathrm{~cm}$ and minimizes respiratory loss (11).

In the article entitled "Re-Assessment of Intentional Extended Segmentectomy for Clinical T1aN0 NonSmall Cell Lung Cancer" published in 2016, authors have compared the outcomes of patients treated with intentional extended segmentectomy and lobectomy in stage IA NSCLC patients (12). Primary endpoint of this study was to assess the long-term survival and the risk factors of local recurrence additionally comparing segment specific results. From 547 cT1aN0 NSCLC operated patients, 237 individuals met eligibility criteria, 164 were treated with segmentectomy and 73 with lobectomy. Follow-up lasted for at least 5 years. Based on thin-section CT scan, patients were divided according to the consolidation to maximum diameter ratio (C/T) (13). In the group of patients with $\mathrm{C} / \mathrm{T}$ $\leq 0.5$, which included 46 segmentectomies and 1 lobectomy, all patients were diagnosed with adenocarcinoma. In that subgroup, there was no recurrence of the disease. In the second subgroup of patients with a $\mathrm{C} / \mathrm{T}$ ratio $>0.5$, that had a diameter of tumor solid component that exceeds half of the maximum tumor diameter, 118 patients underwent segmentectomy and 72 patients lobectomy. In order to reduce the risk of selection bias, propensity score matched analysis with $1: 1$ ratio was performed. Finally, they selected two, 59-patient groups. In the unmatched groups, 5- and 10 -year survival rates were $86.4 \%$ and $70.0 \%$ for segmentectomy and $93.0 \%$ and $78.3 \%$ for lobectomy respectively (log-rank $\mathrm{P}=0.123$ ). However, statistically significant differences in the 5- and 10-year disease-free survival rates were observed for segmentectomies $75.0 \%$ and $61.8 \%$ for lobectomies $90.3 \%$ and $76.4 \%$ (log-rank $\mathrm{P}=0.038$ ). The 5 -year disease-free survival was $76.3 \%$ in segmentectomy and $91.5 \%$ in lobectomy group without significant difference (log-rank $\mathrm{P}=0.082)$. Interestingly, in segment specific analysis of local or regional recurrence it was shown that for superior segments of the lower lobe (S6) the proportion of local or regional recurrences was similar in comparison to lower lobe lobectomies. Segmentectomies of the left upper lobe segments also have a local recurrence rate comparable to upper lobectomies. There was a significantly higher incidence of local and regional recurrences in the basal segments (37\%), compared to lower lobectomies $(\mathrm{P}=0.005)$. The authors reported no significant difference in local-regional recurrence-free survival of right upper segmentectomies compared to right upper lobectomies $(\mathrm{P}=0.075)$.

The authors conclude that for the radiologically invasive form of lung cancer in the clinical stage of cT1aN0Mx located in the right upper lobe or in the basal segments, the decision to perform the intentional segmentectomy must be carefully considered. For superior segments of the lower lobes on both sides and upper left lobe segments, segmentectomy may be a preferred option with outcomes comparable to lobectomy (12).

In conclusion, it is inevitable that waiting for the results of randomized trials, we have to undertake our clinical decisions on well-designed retrospective studies. Conclusions based on the presented paper can have a very significant impact on daily clinical practice. Firstly, patients with clinically indolent tumors with diameter up to $2 \mathrm{~cm}$ 
with ground-glass opacity character or part-solid nodules with solid component up to $50 \%$ of tumor diameter may be good candidates for sublobar resection because such patients are experiencing excellent, disease-free survival. Secondly, in patients with a solid or part solid nodule exceeding $50 \%$ of its diameter due to the more aggressive character of the neoplasm, basal segmentectomies could not be considered as an alternative for lobectomies. Due to good long-term results of segmentectomies of the superior segments of the both sides and segments of the left upper lobe, such patients may be eligible for sublobar resection. All these assumptions however still should be considered very cautiously due to limited credibility of retrospective studies.

\section{Acknowledgements}

The author wishes to thank Witold Rzyman, $\mathrm{MD}, \mathrm{PhD}$, for assistance in preparing the manuscript.

\section{Footnote}

Conflicts of interest: The author has no conflicts of interest to declare.

\section{References}

1. Vansteenkiste J, Crinò L, Dooms C, et al. 2nd ESMO Consensus Conference on Lung Cancer: early-stage nonsmall-cell lung cancer consensus on diagnosis, treatment and follow-up. Ann Oncol 2014;25:1462-74.

2. Ginsberg RJ, Rubinstein LV. Randomized trial of lobectomy versus limited resection for T1 N0 non-small cell lung cancer. Lung Cancer Study Group. Ann Thorac Surg 1995;60:615-22; discussion 622-3.

3. Tsutani Y, Miyata Y, Nakayama H, et al. Segmentectomy for clinical stage IA lung adenocarcinoma showing solid dominance on radiology. Eur J Cardiothorac Surg 2014;46:637-42.

4. Dziedzic R, Zurek W, Marjanski T, et al. Stage I nonsmall-cell lung cancer: long-term results of lobectomy

Cite this article as: Dziedzic R. The role of sublobar resections in the treatment of early stage non-small cell lung cancer-still awaiting evidence. J Thorac Dis 2017;9(11):4146-4148. doi: 10.21037/jtd.2017.10.115 versus sublobar resection from the Polish National Lung Cancer Registry. Eur J Cardiothorac Surg 2017;52:363-9.

5. Altorki NK, Kamel MK, Narula N, et al. Anatomical Segmentectomy and Wedge Resections Are Associated with Comparable Outcomes for Patients with Small cT1N0 Non-Small Cell Lung Cancer. J Thorac Oncol 2016;11:1984-92.

6. Wolf AS, Swanson SJ, Yip R, et al. The Impact of Margins on Outcomes After Wedge Resection for Stage I Non-Small Cell Lung Cancer. Ann Thorac Surg 2017;104:1171-8.

7. Sawabata N, Maeda H, Matsumura A, et al. Clinical implications of the margin cytology findings and margin/ tumor size ratio in patients who underwent pulmonary excision for peripheral non-small cell lung cancer. Surg Today 2012;42:238-44.

8. Nakamura K, Saji H, Nakajima R, et al. A phase III randomized trial of lobectomy versus limited resection for small-sized peripheral non-small cell lung cancer (JCOG0802/WJOG4607L). Jpn J Clin Oncol 2010;40:271-4.

9. Kohman LJ, Gu L, Altorki N, et al. Biopsy first: Lessons learned from Cancer and Leukemia Group B (CALGB) 140503. J Thorac Cardiovasc Surg 2017;153:1592-7.

10. Okada M, Yoshikawa K, Hatta T, et al. Is segmentectomy with lymph node assessment an alternative to lobectomy for non-small cell lung cancer of $2 \mathrm{~cm}$ or smaller? Ann Thorac Surg 2001;71:956-60; discussion 961.

11. Yoshikawa K, Tsubota N, Kodama K, et al. Prospective study of extended segmentectomy for small lung tumors: the final report. Ann Thorac Surg 2002;73:1055-8; discussion 1058-9.

12. Nishio W, Yoshimura M, Maniwa Y, et al. Re-Assessment of Intentional Extended Segmentectomy for Clinical T1aN0 Non-Small Cell Lung Cancer. Ann Thorac Surg 2016;102:1702-10.

13. Suzuki K, Koike T, Asakawa T, Kusumoto M, et al. A prospective radiological study of thin-section computed tomography to predict pathological noninvasiveness in peripheral clinical IA lung cancer (Japan Clinical Oncology Group 0201). J Thorac Oncol 2011;6:751-6. 